\title{
Rivalités linguistiques et efforts de promotion du français à l'Unesco de 1945 à 1970
}

Chloé Maurel

\section{(2) OpenEdition \\ 1 Journals}

\section{Édition électronique}

URL : https://journals.openedition.org/dhfles/105

DOI : $10.4000 /$ dhfles. 105

ISSN : 2221-4038

Éditeur

Société Internationale pour l'Histoire du Français Langue Étrangère ou Seconde

Édition imprimée

Date de publication : 1 janvier 2008

Pagination : $77-100$

ISSN : 0992-7654

\section{Référence électronique}

Chloé Maurel, «Rivalités linguistiques et efforts de promotion du français à l'Unesco de 1945 à 1970 », Documents pour l'histoire du français langue étrangère ou seconde [En ligne], 40/41 | 2008, mis en ligne le 17 décembre 2010, consulté le 27 mai 2021. URL : http://journals.openedition.org/dhfles/105 ; DOI : https://doi.org/10.4000/dhfles.105

Ce document a été généré automatiquement le 27 mai 2021.

(C) SIHFLES 


\title{
Rivalités linguistiques et efforts de promotion du français à l'Unesco de 1945 à 1970
}

\author{
Chloé Maurel
}

1 Au lendemain de la Seconde Guerre mondiale, dans le cadre de la mise en place du système des Nations Unies est créée l'Unesco, Organisation des Nations Unies pour l'éducation, la science et la culture. Comme l'institution qui l'avait précédée, l'Institut international de coopération intellectuelle (IICI) qui avait fonctionné dans l'entre-deuxguerres sous l'égide de la Société des Nations (SDN), l'Unesco est installée à Paris. Elle est dotée de deux langues officielles, l'anglais et le français. Dès le début, une concurrence se développe entre ces deux langues. Deux «clans », groupes d'États aux intérêts linguistiques et culturels divergents se forment: le «clan latin » autour de la France, et le «clan anglo-saxon» autour des États-Unis et du Royaume-Uni, selon la terminologie employée dans les correspondances diplomatiques de l'époque. La nationalité des dirigeants, la langue utilisée dans les nombreuses réunions, conférences, et dans les divers projets qui sont menés sur le terrain, ainsi que le choix du lieu où ils se déroulent, deviennent l'objet de vives luttes d'influence qui se déploient en marge des enjeux officiels de l'organisation, avec comme enjeu le rayonnement de la francophonie ou l'hégémonie de l'anglophonie.

2 Pourquoi et comment l'Unesco, instituée pour rassembler harmonieusement les peuples de différentes langues et cultures, est-elle devenue une arène où se sont au contraire déroulées de féroces luttes entre factions linguistiques opposées ? En quoi la rivalité entre ces deux «clans» a-t-elle pris aussi une dimension politique et économique ? Comment a évolué l'équilibre des forces entre le français et l'anglais au sein de l'Unesco de 1945 à 1970 ?

3 La perspective chronologique permettra de mettre en évidence les étapes de l'évolution qui se dessine progressivement: si de 1945 à 1948, l'organisation est divisée entre l'influence anglo-saxonne et l'influence francophone, cette tendance s'infléchit de 1948 à 1952 vers une influence francophone croissante, et, après une tentative de 
restauration de l'influence anglo-saxonne de 1953 à 1958, l'influence francophone gagne du terrain tout au long des années 1960 de sorte que vers 1970 la langue française et les conceptions culturelles francophones bénéficient d'une place prépondérante à l'Unesco.

\section{5-1948. L'apparition précoce d'un clivage entre " clan latin » et « clan anglo-saxon »}

Dans ses premières années, entre 1945 et 1947, l'Unesco est divisée, sur le plan linguistique et culturel, entre l'influence anglo-saxonne et l'influence francophone.

Les premières assemblées et réunions de l'Unesco naissante, en 1945-1946, sont marquées par la participation d'intellectuels français tels Léon Blum, Paul Rivet, Jacques Maritain, Lucien Febvre, René Cassin, Frédéric Joliot-Curie, François Mauriac, dans l'esprit de l'ancien Institut international de coopération intellectuelle (IICI) de l'entre-deux-guerres, qui s'était caractérisé par une conception classique, élitiste, franco-centrée, de la culture ${ }^{1}$.

6 Pourtant, par rapport à la période de l'IICI, la création de l'Unesco marque une véritable rupture : alors que, durant l'entre-deux-guerres, les États-Unis avaient refusé de participer à la SDN et à ses institutions culturelles et parmi elles l'IICI, la Seconde Guerre mondiale a déclenché dans l'esprit du gouvernement américain une prise de conscience de l'importance pour les États-Unis de s'impliquer désormais davantage dans les affaires politiques et culturelles internationales. Ainsi, dès la création de l'Unesco, les États-Unis y adhèrent et le gouvernement américain se montre très soucieux d'exercer une grande influence sur ce nouvel organisme culturel.

7 Les États-Unis s'efforcent notamment d'orienter les programmes et activités de l'Unesco vers une action culturelle qui se fasse au moyen des mass media (radio, télévision, presse à grand tirage, cinéma), s'opposant en cela au caractère intellectuel, élitiste et européen qui avait caractérisé l'action de l'IICI. En promouvant l'usage des médias de masse, les États-Unis espèrent aussi utiliser l'Unesco comme un vecteur pour diffuser dans le monde entier leur langue, leur idéologie, et leurs produits culturels². L'Unesco présente donc pour les États-Unis un triple intérêt: à la fois culturel, car permettant d'asseoir l'hégémonie de la langue anglaise dans le monde; politique, dans le cadre de la guerre froide naissante donc de l'opposition idéologique entre deux blocs; et économique, l'Unesco permettant aux États-Unis de s'ouvrir «de nouveaux marchés et des possibilités d'investissement outre-mer ", comme l'affirme Walter Laves, directeur général adjoint de l'Unesco de 1946 à 1950, dans un discours devant des représentants des milieux d'affaires américains ${ }^{3}$.

8 Le gouvernement français, quant à lui, est également très intéressé par l'Unesco, dans laquelle il voit un moyen de développer le rayonnement de la culture et de la langue française dans le monde. C'est pourquoi, lors de la conférence constitutive de l'Unesco, le gouvernement français insiste, par la voix de son représentant, Léon Blum, pour obtenir la localisation du siège de l'organisme à Paris, contre la préférence des ÉtatsUnis, qui préconisaient plutôt une ville du Danemark, de Hollande ou de Norvège. Finalement un accord secret est passé entre Français et Américains: le siège de l'Unesco sera à Paris, mais en échange le poste de directeur général (DG) de l'Unesco ne serait jamais accordé à un Français, et le premier DG à être élu sera un Anglo-Saxon" 
Cependant, le candidat américain envisagé par les États-Unis, le poète et bibliothécaire du Congrès Archibald MacLeish, que la France s'est engagée à soutenir, se désiste au dernier moment $^{5}$. Les États-Unis ne parvenant pas à lui trouver un remplaçant compétent, c'est finalement le candidat britannique qui est présenté, bien qu'il soit loin de faire l'unanimité au gouvernement américain en raison de son caractère fantasque et de sa sensibilité de gauche : il s'agit du biologiste Julian Huxley, frère de l'écrivain Aldous Huxley. Il est élu de justesse ${ }^{6}$. Même s'il n'est pas américain et si sa personnalité est peu appréciée du gouvernement américain, il s'agit néanmoins d'un Anglo-Saxon. De plus, d'autres gages sont encore donnés aux États-Unis, comme par exemple la désignation d'un «directeur-général adjoint pour l'administration », poste attribué à l'Américain Walter Laves, agent du département d'État.

10 Pendant tout le mandat de Julian Huxley, Walter Laves joue un rôle majeur à l'Unesco, prenant en charge l'essentiel des décisions administratives et décidant d'une grande partie des orientations importantes de l'organisation. En effet, le scientifique Julien Huxley, n'ayant pas beaucoup d'inclination pour les tâches administratives, lui laisse carte blanche sur de nombreux points. Walter Laves apparait donc comme un DG officieux. Ainsi, en 1947, le ministre français des Affaires étrangères, Georges Bidault, observe avec préoccupation que Walter Laves est «le maître absolu des finances, de l'administration et même du recrutement de l'Unesco ». Il déplore : "aux États-Unis même, un service spécial du département d'État envoie sans arrêt de volumineux mémorandums à l'hôtel Majestic [bâtiment initial de l'Unesco à Paris] et aux États membres qui se demandent, à juste titre, si le véritable directeur général n'est pas plutôt à Washington qu'à Paris ». Georges Bidault observe avec inquiétude à quel point l'Unesco est rapidement devenue " une institution soumise à l'influence américaine " ${ }^{7}$. En effet, les membres de la délégation américaine à l'Unesco sont choisis directement par le département d'État, et le président de cette délégation, William Benton, est secrétaire d'État adjoint des États-Unis ${ }^{8}$. La France obtient cependant, quelques mois plus tard, à titre de contre-poids, la nomination d'un second DG adjoint : à côté de celui préposé à l'administration, apparaît un « DG adjoint pour la culture », le Français Jean Thomas. De formation littéraire, normalien, ancien enseignant à l'École normale supérieure et auteur d'ouvrages sur la philosophie des Lumières ${ }^{9}$, Jean Thomas s'inscrit dans la tradition intellectuelle, élitiste et franco-centrée qui avait caractérisé l'IICI. "Pour ceux qui l'ont connu, il était et restera l'incarnation du ' $C$ ' dans le sigle Unesco ", écrit à son sujet son ancien collègue Émile Delavenay ${ }^{10}$. La France réussit donc à négocier un relatif rééquilibrage des nationalités au sein de l'équipe dirigeante.

11 Durant tout le mandat de Julian Huxley, l'Unesco est le théâtre d'importants conflits idéologiques et linguistiques entre la France et les États-Unis. Dès 1947, on voit, dans les archives confidentielles, émerger les termes de " clan anglo-saxon » et de " clan latin », deux groupes de pression opposés représentés chacun par une langue : l'anglais et le français ${ }^{11}$. Les archives diplomatiques françaises révèlent la détermination des diplomates français à résister au "clan anglo-saxon", à l'«impérialisme culturel américain », et à assurer le "maintien de la pensée et de la langue française » à l'Unesco ${ }^{12}$.

12 En 1947, les Anglo-Saxons sont cependant majoritaires parmi le haut personnel de l'Unesco. Julian Huxley observe ainsi cette année-là dans une lettre à Walter Laves : «beaucoup d'autres nations sont mécontentes de ce qu'elles considèrent comme une influence injustifiée des Anglo-Saxons, et particulièrement des Américains ${ }^{13}$. Le 
gouvernement français fait tout son possible pour favoriser le recrutement de Français ou de "personnalités étrangères ayant un bon usage de notre langue et favorables à nos idées et à notre culture ", comme l'exprime Georges Bidault, mais ces efforts demeurent peu fructueux. Ainsi, en août 1947, Bidault regrette qu'aucun grand intellectuel français n'ait reçu de poste important à l'Unesco : «L'Hôtel Majestic [...] est resté une citadelle anglo-saxonne au cœur de Paris. Aucun emploi important en dehors de quelques postes administratifs n'a été offert à un Français. $»^{14}$

13 L'article « La France à l'Unesco » publié par le sociologue, historien et géographe André Siegfried en première page du Figaro en mars 1948 est révélateur de la rivalité linguistique entre le français et l'anglais qui se développe à l'Unesco, et aussi de la volonté de la France d'utiliser l'Unesco pour maintenir et développer son rayonnement culturel et linguistique dans le monde : selon lui, face à l'ambition des États-Unis de faire de l'Unesco une "machine » qui enseignerait le "way of life américain » en promouvant la libre circulation de l'information, il existerait à l'Unesco une autre conception, «qui relève au fond de la latinité et dont nous [les Français] nous sommes trouvés, sans l'avoir directement cherché, les représentants les plus actifs et les plus influents ». Cette conception latine se caractériserait par la défense des valeurs de "l'humanité ", et par "une résistance latente contre cette forme trop évidente de puissance que représente aujourd'hui la presse américaine, la radio américaine, le film américain, l'agence d'information américaine, sans oublier ces fameux comic-strips qui se sont répandus sur le monde entier ». Critiquant la conception américaine de la culture, André Siegfried lui oppose la culture classique française. Il présente la France comme «le leader naturel d'une foule de pays soucieux de freiner une trop rapide américanisation de la planète ». Il inclut dans ce groupe «l'Amérique latine, les pays méditerranéens, le proche Orient, l'Inde ». Il s'efforce de justifier la place de premier plan de la France à la tête de ce mouvement : « je n'exagère certainement pas en disant que la France est le leader désigné de pareil mouvement, car elle le représente [...] avec éclat. [...]. Tous ceux qui voyagent constatent, en effet, que notre prestige culturel est intact. [...] La vitalité spirituelle de la France est intacte. Notre délégation à Mexico [à l'occasion de la $2^{\mathrm{e}}$ conférence générale de l'Unesco, en 1947] était brillante [...]. Nous voyons là en quoi nous avons conservé sur le monde, du moins sur certaines parties du monde, une incontestable emprise. $»^{15}$ Ces affirmations d'autosatisfaction témoignent en fait du besoin des Français de se rassurer et révèlent l'inquiétude de la France qui se rend compte du progressif déclin de son prestige linguistique et culturel dans le monde au profit de la culture anglo-saxonne et en particulier américaine.

14 Cependant, si durant ces premières années la langue anglaise et les conceptions culturelles anglo-saxonnes rivalisent sérieusement avec l'influence française au sein de l'Unesco, les années suivantes amènent une évolution.

\section{8-1952. Une influence « latine » croissante}

De la fin 1948 à la fin 1952, l'Unesco évolue vers une tendance plus « latine ». Aussi bien dans son fonctionnement administratif que dans les actions menées dans ses États membres, un regain de la francophonie s'affirme.

En décembre 1948, un nouveau directeur général est élu en rempla-cement de Julian Huxley: le Mexicain Jaime Torres Bodet. Ancien instituteur et bibliothécaire, poète, diplomate et homme politique, il allie en lui des qualités qui en font apparemment 
l'homme parfait pour ce poste. De 1943 à 1946, il a été ministre de l'Éducation publique du Mexique et a organisé une vaste campagne nationale d'alphabétisation, efficace et populaire ${ }^{16}$.

17 Surtout, il est très apprécié du gouvernement français, pour son profil d'intellectuel francophone et francophile : sa mère étant française, il parle couramment le français, et utilise volontiers cette langue. Il apprécie beaucoup la culture française, s'intéresse à l'histoire de France et est lié aux milieux littéraires français. En outre, dans les années 1930, il a participé à plusieurs réunions de l'IICI à Paris. Admirateur de cet organisme qui était d'inspiration française, il le revendique ouvertement comme un modèle pour l'Unesco ${ }^{17}$, alors que lors de la création de l'Unesco les Anglo-Saxons avaient présenté l'IICI comme un contre-modèle à ne pas imiter. Fidèle à l'esprit de l'IICI, Torres Bodet se montre extrêmement soucieux de faire participer les élites littéraires à l'action de l'Unesco. Cette orientation intellectuelle qu'il entend donner à l'Unesco correspond bien à celle prônée par la France et s'oppose à celle préconisée par les États-Unis, fondée sur les mass media et la diffusion d'une culture de masse.

$18 \mathrm{Au}$ sein de l'administration de l'Unesco, Torres Bodet, de par son profil intellectuel et sa parfaite maîtrise du français, se lie beaucoup plus aisément avec les fonctionnaires français ou en tout cas francophones qu'avec les Anglo-Saxons. Il se plait à entamer des conversations littéraires avec des écrivains français travaillant pour l'Unesco comme Roger Caillois ou Philippe Soupault ${ }^{18}$. En outre, à peine élu directeur général, il nomme immédiatement le Français René Maheu directeur de son cabinet. Celui-ci raconte : « il y avait eu entre nous une sympathie immédiate et sa conception humaniste de la mission de l'Unesco rejoignait tout à fait mes idées personnelles ${ }^{19}$. C'est le début d'une étroite collaboration entre les deux hommes. Torres Bodet offre ainsi à Maheu un tremplin qui lui permettra une quinzaine d'années plus tard de devenir DG à son tour.

19 Le fait que Torres Bodet s'exprime couramment en français contribue beaucoup à conférer une place prépondérante au sein de l'administration de l'Unesco au personnel français, et donc aux idées françaises. Ainsi, durant son mandat, l'influence de l'Américain Walter Laves décline beaucoup au profit de celle d'un petit groupe de Français comme Jacques Havet, Jean Thomas, Jean-Jacques Mayoux (ancien directeur de l'IICI), et surtout René Maheu. Torres Bodet travaille aussi en lien avec les ministres français des Affaires étrangères de l'époque, Georges Bidault et Robert Schuman ${ }^{20}$.

Pendant ces années-là, plusieurs éminents intellectuels français ou francophones, comme les ethnologues Claude Lévi-Strauss, Alfred Métraux, Michel Leiris, GeorgesHenri Rivière, Georges Balandier, ou encore les cinéastes documentaristes Chris Marker et Jean Rouch, accomplissent des missions pour l'Unesco. C'est souvent par des réseaux de connaissances que ces intellectuels francophones entrent en contact avec l'Unesco et y obtiennent des postes ou, plus fréquemment, des missions ponctuelles. C'est le cas de Claude Lévi-Strauss ${ }^{21}$ : ainsi, en 1951, son confrère Alfred Métraux le choisit pour rédiger une des publications de la série de brochures de l'Unesco sur le problème du racisme. Si la quasi-totalité des publications de cette série sont rapidement tombées dans l'oubli, celle de Claude Lévi-Strauss, Race et Histoire ${ }^{22}$ (une des seules d'ailleurs à être écrite par un Français et non par un Anglo-Saxon) deviendra célèbre. Ces réseaux permettent le recrutement et la participation d'une portion croissante de francophones à l'Unesco. À l'inverse, plusieurs Anglo-Saxons quittent l'Unesco au cours de ces années, leurs contrats n'étant pas renouvelés. 
21 Ainsi le mandat de Torres Bodet, qui dure jusqu'en 1952, entraîne un accroissement de l'influence « latine » et en particulier de l'influence francophone, et corollairement un déclin de l'influence anglo-saxonne. Cette évolution est observée avec inquiétude par les diplomates anglo-saxons. Dès le début du mandat de Torres Bodet, le physicien américain Arthur Compton, prix Nobel de physique 1927, employé par l'Unesco, observe dans un rapport confidentiel au département d'État américain, que le groupe des pays qu'il désigne comme "européens latins", au nombre desquels il place notamment la France, la Belgique, le Luxembourg, l'Italie, la Suisse et la Grèce, « est très influencé par la direction intellectuelle française et ne partage généralement pas l'insistance mise par les États-Unis sur la participation des 'masses' à l'Unesco ». Il observe corollairement la perte d'influence du groupe des États menés par les ÉtatsUnis, auxquels se rattachent le Royaume-Uni et les membres ou anciens membres du Commonwealth (Nouvelle-Zélande, Australie, Canada, Afrique du Sud et Inde), ainsi que les États scandinaves (Danemark et Norvège) ${ }^{23}$.

De même, en 1950, un diplomate britannique estime, dans un rapport confidentiel adressé au Foreign Office, qu'au sein de l'Unesco « il semble y avoir un certain retour à l'esprit de l'IICI de l'avant-guerre, et ce retour est particulièrement inspiré par les Français, auxquels le DG actuel accorde une très grande attention ». Ce diplomate s'inquiète de « la croissance de l'influence française par rapport à l'influence angloaméricaine à l'Unesco", jugeant que les hauts fonctionnaires de l'Unesco sont désormais tous « de profil culturel principalement français ». Il précise : « le DG luimême se considère comme français de cœur $»^{24}$. La même année, le conseiller du département d'État sur les affaires de l'Unesco, Kenneth Holland, observe que l'antagonisme entre Français et Américains au Secrétariat se répercute nettement sur les activités de l'organisation, et que les conceptions françaises sont en passe de l'emporter sur les conceptions américaines ${ }^{25}$.

23 Au cours du mandat de Torres Bodet, le «clan latin » non seulement se renforce mais aussi s'élargit : plusieurs États arabes et latino-américains s'y joignent. La France utilise notamment la conférence générale de décembre 1948, tenue à Beyrouth, comme moyen de resserrer ses liens avec le Liban et avec les pays arabes comme l'Égypte, ainsi qu'en témoignent les articles très francophiles parus à ce sujet dans le journal francophone Le Journal d'Égypte ${ }^{26}$. Le choix de Beyrouth pour cette conférence (au cours de laquelle est élu Torres Bodet) est le résultat des efforts déployés par Jean Maroun, vicaire patriarcal maronite à Paris et membre de la délégation libanaise à l'Unesco, imprégné de culture classique française. L'ambassade américaine à Beyrouth note avec inquiétude dans un rapport confidentiel au département d'État américain que Jean Maroun, titulaire d'un doctorat de la Sorbonne, serait «anti-américain» et "d'orientation presque complètement pro-française $»^{27}$. À l'occasion de la conférence de Beyrouth, la France organise d'importantes manifestations culturelles dans cette ville pour accroître son prestige et son rayonnement culturel auprès de la population de cet ancien territoire sous mandat français ${ }^{28}$. Dans les années qui suivent, le choix des pays où se déroulent les conférences générales de l'Unesco est révélateur : outre le Liban (décembre 1948), c'est l'Italie (Florence, 1950), et à trois reprises la France (Paris, 1949, 1951 et 1952), ce qui montre bien l'orientation « latine » de l'Unesco dans ces années.

Enfin, au cours de cette période, le clan latin s'enrichit d'un nouvel allié: la Yougoslavie, qui adhère à l'Unesco en mars 1950. Son représentant, M. Ribnikar, est ouvertement francophile; un rapport confidentiel du Quai d'Orsay de l'époque observe 
ainsi, en se réjouissant: «M. Ribnikar nourrit à l'égard de notre pays, qu'il connaît bien, de sincères sentiments d'amitié et possède parfaitement notre langue ${ }^{29}$.

Par ailleurs, la gestion de l'administration de l'Unesco par l'Américain Walter Laves est à partir du début du mandat de Torres Bodet contestée de plus en plus nettement au sein de l'Unesco, à tel point que le département d'État s'inquiète de "la marée croissante d'un sentiment anti-américain » dans l'administration de l'Unesco ${ }^{30}$. En décembre 1949, une note confidentielle du ministère des Affaires étrangères français exprime l'opposition de la France au renouvellement du contrat de Walter Laves à son poste. Significativement, les arguments avancés sont les suivants : « connaissant mal la langue française, peu au fait des modes de penser européens, M. Laves a exercé [...] une action tendant à faire prévaloir, au sein de l'organisation, des conceptions administratives trop étroitement américanistes $\|^{31}$. En janvier 1950, Laves lui-même se considère comme «le principal rempart des intérêts américains dans l'organisation ", et confie à son gouvernement qu'il s'y sent mis en minorité, menacé3 ${ }^{32}$. Il perd effectivement son poste dans les semaines qui suivent. Sous Torres Bodet, le recrutement de personnes francophones aux postes à responsabilité s'accroît beaucoup, au détriment des Anglo-Saxons. Ainsi, en 1950, la nationalité majoritaire parmi les postes de hauts fonctionnaires est désormais la nationalité française : 60 de ces postes sont occupés par des Français, contre 51 par des Britanniques et 45 par des Américains $^{33}$. C'est un véritable retournement par rapport aux premières années.

L'opposition entre clan latin et clan anglo-saxon au sein du Secrétariat se manifeste très clairement à cette époque à travers les rivalités et les inimitiés entre personnel francophone et personnel anglo-saxon. En particulier on peut relever, dans les différentes divisions de l'Organisation, plusieurs exemples de vives tensions entre collègues de travail américains et françaiis ${ }^{34}$. Elles ont pour fondement des divergences à la fois linguistiques, culturelles et conceptuelles. En 1951 et 1952, le conseiller du département d'État sur les affaires de l'Unesco, Kenneth Holland, transmet à plusieurs reprises au département d'État, dans ses rapports confidentiels hebdomadaires, l'insatisfaction croissante de nombreux Américains employés par l'Unesco, insatisfaction due au sentiment que le directeur général ne les écoute pas et leur préfère le personnel français ${ }^{35}$.

La langue employée au sein du «Secrétariat» (c'est-à-dire de l'administration de l'Unesco) apparaît ainsi sans ambiguïté comme un vecteur majeur d'influence culturelle, idéologique, et politique. Si sous le mandat de Julian Huxley l'anglais a prédominé, sous celui de Torres Bodet la tendance s'inverse au profit du français, langue que celui-ci utilise systématiquement, au détriment de l'anglais, dans les réunions, dans ses conversations avec le personnel et dans ses notes et correspondances. Les Américains observent et déplorent que l'emploi du français par le directeur général « a considérablement modifié le caractère » des réunions, et constate que, de ce fait, le personnel francophone joue désormais un rôle beaucoup plus important que par le passé ${ }^{36}$. Le département d'État américain observe et dénonce également le fait que sous Torres Bodet l'un des critères majeurs, bien qu'officieux, pour le recrutement du personnel, est la maîtrise du français. En réponse au favoritisme dont ils estiment que les Français bénéficient, les Américains dénigrent les documents rédigés en français, affirmant qu'ils seraient moins rigoureux et plus verbeux que ceux rédigés en anglais ${ }^{37}$. 
ussi dans la réalisation des actions de l'Unesco sur le terrain qu'on observe de fortes rivalités entre le français et l'anglais. Ces rivalités opposent notamment le département d'État américain au Quai d'Orsay au sujet de l'emploi de la langue d'enseignement dans le programme d'éducation UNESCO-UNRWA ${ }^{38}$ au Proche-Orient, programme visant à apporter une enseignement scolaire aux enfants palestiniens réfugiés dans des camps au Liban, en Syrie, en Jordanie ou dans la bande de Gaza : ainsi, en 1951 la Direction générale des affaires culturelles française se préoccupe vivement du maintien de la langue française «sur un pied de complète égalité » avec la langue anglaise dans ce programme. Cette compétition linguistique entre français et anglais pour l'éducation des enfants du programme UNESCO-UNRWA se poursuivra de nombreuses années durant ${ }^{39}$.

de même au sujet du choix de la langue d'enseignement au Centre d'éducation de base pour les États arabes (Arab States Fundamental Education Center, ASFEC) que l'Unesco crée en Égypte en 1952: Georges Bidault charge l'ambassadeur français au Caire de s'efforcer de "provoquer le recrutement du plus grand nombre possible de professeurs et d'instructeurs français » pour contrer la tradition anglophone répandue dans ce pays ${ }^{40}$.

30

Enfin, le " projet-pilote » d'« éducation de base » mené par l'Unesco sur l'île d'Haïti de 1946 au début des années 1950 est également l'occasion de vives rivalités linguistiques entre l'anglais et le français. Dans le cadre de ce projet, centré sur les habitants d'une vallée misérable et enclavée d'Haïti, l'Unesco soutient des efforts de linguistes pour réaliser une transcription orthographique du créole haïtien, langue vernaculaire très proche du français. Des controverses ne tardent pas à éclater entre experts français et experts américains, sur la manière de transcrire cette langue, les uns préconisant une transcription sur le modèle du français, les autres sur le modèle de l'anglais. Des rivalités s'affirment aussi sur le plan pédagogique, au sujet de la méthode d'éducation qu'il s'agit de mettre en place: doit-elle s'inspirer des méthodes pédagogiques françaises ou anglo-saxonnes? Et surtout, en quelle langue doit être dispensé cet enseignement? À travers les débats et oppositions qui éclosent, on saisit une rivalité politique entre l'influence de la langue anglaise et celle de la langue française ${ }^{41}$. Elle est d'autant plus aiguë qu'Haïti, l'ancienne colonie française de Saint-Domingue, qui a acquis son indépendance en 1804 , est alors un pays où la culture française est historiquement importante et très enracinée. Or, située non loin des États-Unis, à proximité des côtes de Floride (à environ 1000 kilomètres de Miami), Haïti constitue pour les États-Unis un enjeu stratégique non négligeable. Au fil du déroulement de ce projet, l'Unesco s'inquiète de la dimension politique de ces controverses, d'autant plus que les Haïtiens sont eux aussi divisés sur ces questions. Les relations tumultueuses pendant ces années entre l'Unesco, le gouvernement haïtien et l'ambassade américaine de Port-au-Prince, témoignent de ces difficultés. L'Unesco finira vers 1954 par abandonner discrètement ce laborieux projet qui a abouti à de piètres résultats ${ }^{42}$.

$31 \mathrm{Au}$ terme de ces années durant lesquelles l'influence francophone se renforce à l'Unesco, les gouvernements des États-Unis et du Royaume-Uni changent d'attitude à l'égard de l'Unesco : déçus d'avoir échoué à y faire triompher la culture anglo-saxonne ils se désengagent de cette organisation ${ }^{43}$. Observant que l'Unesco ne leur offre aucun profit tangible sur le plan politique, économique, idéologique, culturel, les États-Unis, en plaidant pour une limitation du budget de l'Unesco, s'efforcent désormais de réduire l'étendue de l'action de cette organisation et de freiner son intervention directe dans 
les États membres, intervention qu'ils perçoivent désormais comme une concurrence par rapport à leurs propres programmes bilatéraux de partenariat avec ces pays. Même l'élection d'un DG américain, Luther Evans, en 1953 pour succéder à Torres Bodet, ne fait pas changer d'avis le gouvernement américain ${ }^{44}$.

Ainsi, la personnalité du deuxième DG, Torres Bodet, ainsi que le développement de réseaux linguistiques et culturels actifs aussi bien parmi le personnel de l'Unesco que dans les cercles diplomatiques permet en quelques années à l'influence francophone de gagner du terrain, et d'apparaître dominante au moment où Torres Bodet quitte l'Unesco fin 1952.

\section{3-1958. Une certaine perte de terrain du « clan latin » par rapport au « clan anglo-saxon »}

La période suivante, de 1953 à 1958, correspond à des tentatives de restauration de l'influence anglo-saxonne à l'Unesco.

Le nouveau DG, le Texan Luther Evans, homme pragmatique et peu porté sur les affaires intellectuelles, tranche nettement par sa personnalité et son langage avec ses collaborateurs francophones, qui forment un groupe soudé par leur hostilité envers lui. Ainsi, l'ancien fonctionnaire français Émile Delavenay évoque Luther Evans de cette manière très péjorative dans ses mémoires : «De petite taille, trapu, mâchoire carrée de bouledogue, verbe haut et souvent vulgaire, la voix aigüe, Evans devait souffrir durant ses années à la tête de l'Unesco, d'un complexe d'infériorité culturelle face à des hommes comme Jean Thomas ou René Maheu. Mais il compensait en agressivité ce sentiment d'appartenir à une civilisation relativement fruste $»^{45}$.

Un véritable «choc culturel» se manifeste entre Luther Evans et les fonctionnaires francophones, comme le laissent percevoir les correspondances internes ainsi que les rapports confidentiels écrits par des francophones. Émile Delavenay témoigne de l'opposition nette entre Luther Evans et son adjoint le Français Jean Thomas lors de ces réunions : «Dans ces moments-là nous frémissions du choc de deux cultures, de deux conceptions des relations humaines $»^{46}$. Comme l'exprime un autre ancien fonctionnaire, Gérard Bolla, entre Evans et Thomas, c'est une opposition non seulement de conceptions mais aussi de caractère, entre la «fougue texane» d'Evans et «la prudence européenne et 'normalienne' de Jean Thomas ${ }^{47}$. Les représentants des ÉtatsUnis observent eux aussi ce fossé: ainsi, en 1958, une lettre confidentielle de l'ambassade américaine de Paris note à propos de Luther Evans: "Sa tendance à appeler un chat un chat, son caractère dur et hargneux, son supposé irrespect pour les choses 'Culturelles' avec un grand C [...] ont suscité une rancœur et une haine considérables parmi les Européens de l'Ouest et du Sud $»^{48}$.

Quoi qu'il en soit, avec Luther Evans à la tête de l'Unesco, c'est un retour provisoire à la langue anglaise qui s'effectue: le nouveau DG s'exprime exclusivement dans cette langue et s'entoure de collaborateurs anglophones. "Mieux valait lui parler anglais si l'on voulait être bien compris ", souligne un ancien fonctionnaire français ${ }^{49}$. Dès lors, le fait de ne pas maîtriser l'anglais devient un véritable handicap, qui conduit à la marginalisation des membres du personnel qui ne parlent pas anglais, comme le sociologue français Roger Caillois ${ }^{50}$. Luther Evans se «débarrasse » même du Français René Maheu, qui était alors sous-directeur-général, et qui pourtant parlait couramment 
anglais : il l'envoie à New York en 1955, le nommant représentant de l'Unesco aux Nations Unies pour l'éloigner du centre de décision qu'est le Siège de l'Unesco. Maheu ne pourra revenir de son « exil » comme il le désigne, et réintégrer le siège parisien de l'Unesco qu'après la fin du mandat de Luther Evans, en $1958^{51}$.

Cependant, malgré cette direction américaine de l'Unesco, le «clan latin » demeure très influent au sein de l'Unesco, notamment grâce à l'importance numérique des Français dans le personnel. C'est aussi dans les diverses réunions et conférences internationales organisées par l'Unesco ou en lien avec l'Unesco que la langue française et les conceptions éducatives et culturelles françaises parviennent à se maintenir.

Finalement, si la période 1953-1958 correspond à un certain recul de l'influence francophone à l'Unesco, et à des tentatives de restaurer l'influence anglo-saxonne, ce phénomène demeure limité : réticent à l'égard de la francophonie, Luther Evans n'apporte pas pour autant une nouvelle conception de l'action culturelle de l'Unesco, qui batte en brèche les conceptions développées par des francophones comme Jean Thomas dans les années précédentes. Se concevant lui-même comme un administrateur et non comme un intellectuel, Luther Evans ne laissera pas de réelle marque sur les orientations culturelles de l'Unesco.

\section{Après 1958. Réaffirmation et renforcement de la francophonie à l'Unesco}

De 1958 à 1970, la domination du courant francophone s'affirme de plus en plus nettement. Cela peut s'expliquer à la fois par la personnalité des dirigeants de l'Unesco de cette période, et par la volonté politique de la France gaullienne : en effet, en ce début de Cinquième République, le ministère des Affaires culturelles créé en 1959 et les réseaux diplomatiques français s'emploient activement à accroître le prestige et le rayonnement culturel de la France dans le monde.

En 1958, l'élection de l'Italien Vittorino Veronese au poste de directeur général de l'Unesco accroît l'orientation vers les conceptions « latines », puisque ce dernier, bien que politiquement « atlantiste », donc proche des États-Unis, maîtrise mieux le français que l'anglais, et de ce fait emploie davantage le français dans l'exercice de ses fonctions et accorde une place prioritaire au personnel francophone. Ainsi, René Maheu est immédiatement rappelé au siège à Paris et retrouve le rôle prépondérant qu'il avait dans la direction de l'Unesco. Veronese le nomme directeur général adjoint, à la grande satisfaction du gouvernement français ${ }^{52}$. Pendant le mandat de Veronese, c'est en pratique Maheu qui dirige l'Unesco. Effacé et rapidement dépassé par le poids des responsabilités et la complexité de l'organisation, Veronese démissionne en 1961, laissant à René Maheu l'intérim pendant un an ${ }^{53}$. L'année suivante, en 1962, René Maheu est élu à son tour DG.

Le gouvernement américain était initialement défavorable à l'élection de Maheu, qui va à l'encontre de l'accord secret de 1945 et du règlement tacite selon lequel les chefs des agences de l'ONU ne doivent pas être citoyens du pays dans lequel elles se trouvent ${ }^{54}$. Cependant, devant l'absence de candidat anglo-saxon compétent, les États-Unis et le Royaume-Uni finissent par se rallier à la candidature de Maheu. Le gouvernement britannique est d'ailleurs très sensible au fait que Maheu parle très bien l'anglais et 
connaisse bien l'Angleterre, où il a vécu de 1933 à 1939 en tant qu'attaché culturel à Londres ${ }^{55}$.

C'est le début pour René Maheu d'un long mandat de douze ans, puisqu'il sera réélu en 1968. Non seulement il est le premier DG à accomplir deux mandats successifs, mais il est aussi le premier DG à aller jusqu'au bout de son premier mandat, tous ses prédécesseurs ayant démissionné avant la fin du leur. Sa longue présence à la tête de l'Unesco lui donne l'occasion d'y imposer une influence francophone hégémonique, ce qui suscite l'irritation des gouvernements britannique et américain ${ }^{56}$.

La prépondérance de la langue et des conceptions françaises se répercute dans les actions de l'Unesco sur le terrain, et en particulier dans les pays d'Afrique récemment décolonisés et ayant adhéré à l'Unesco. Ainsi en 1965, une représentante du RoyaumeUni, visitant plusieurs de ces pays d'Afrique, observe une tendance croissante de ces gouvernements à être influencés par «les méthodes, le langage et le système d'administration français ", en particulier au Cameroun, où le Royaume-Uni et la France rivalisent pour leur influence dans ce pays dont la partie orientale est anglophone, et la partie occidentale francophone, et où elle observe que les actions de l'Unesco entraînent une extension de l'influence française dans la partie orientale ${ }^{57}$. De fait, dans les États d'Afrique, dont beaucoup sont des ex-colonies de la France ou du Royaume-Uni, et où l'Unesco organise ou soutient dans les années 1960 d'importants projets éducatifs, le choix de la langue d'enseignement (anglais ou français) pour des millions d'enfants africains constitue un enjeu majeur. René Maheu réussit à imposer le maintien du français dans la plupart des nombreuses écoles et écoles normales qui dans ces années, avec l'aide de l'Unesco, sont créées en Afrique (souvent sur le modèle des «écoles normales » françaises d'ailleurs) ${ }^{58}$.

Des enjeux analogues se déploient aussi en Amérique latine, où, dans le cadre d'un ambitieux projet éducatif mené par l'Unesco sur ce continent, on observe dans les années 1960 une concurrence linguistique entre les experts américains et français envoyés donner des cours à l'université de Sao Paulo. Américains et Français se surveillent mutuellement, rivalisent pour imposer aux étudiants leur propre langue, et par-delà leur langue leurs méthodes de travail et leur manière de pensée, et poussent l'Unesco à nommer davantage d'experts de leur propre nationalités9.

La rivalité entre anglophonie et francophonie se déploie aussi à travers les débats et les conflits au sujet du choix de certains termes ou de la traduction de certains concepts dans l'intitulé de certaines actions de l'Unesco. En effet, l'anglais et le français étant les deux langues officielles de l'Unesco, tous les documents officiels doivent paraître dans les deux versions linguistiques, et l'organigramme de l'Unesco doit aussi apparaître dans les deux langues. Loin d'être anodines, de telles questions de vocabulaire prennent souvent des dimensions politiques. Il en va ainsi du choix du terme "information" obtenu par les Français en 1965 pour l'intitulé d'une nouvelle division administrative de l'Unesco, de préférence au terme «communication» qui renvoie davantage aux communications de masse, et donc aux conceptions américaines ${ }^{60}$. De même, au milieu des années 1960, dans le cadre de la rédaction, sous la houlette de l'Unesco, de l'ouvrage de synthèse Tendances principales de la recherche dans les sciences sociales et humaines, des tensions éclosent au sujet de la traduction en anglais du terme "sciences de l'homme " Faut-il le rendre par "social sciences" ou "human sciences", ou encore "sciences of man "? Cette question de traduction a des enjeux de prestige national. En effet, le terme français de "sciences de l'homme ", remontant aux philosophes français 
des Lumières, est plus flatteur pour la France. Les Français insistent donc pour l'introduction du terme anglais « sciences of man ", calqué sur le terme français ${ }^{61}$.

La rivalité entre anglais et français aboutit aussi parfois à paralyser certains projets de l'Unesco. Ainsi, en 1970, un projet de « lexique notionnel international », lancé par le poète français Yves Bonnefoy et soutenu par l'écrivain Jean d'Ormesson et par René Maheu, et également par le gouvernement français, qui se réjouit que «la part des Français dans [sa] réalisation sera prépondérante », est finalement abandonné à cause de l'opposition des Anglo-Saxons. Avec le recul du temps, le poète Yves Bonnefoy analyse avec amertume : « mon projet est devenu un ballon de football qui a été utilisé par les équipes nationales en conflit $»^{62}$.

47 En 1970, les États-Unis s'inquiètent de plus en plus de l'influence française jugée «considérable» à l'Unesco, en lien avec la forte prépondérance numérique des Français au sein du personnel au détriment des Anglo-Saxons ${ }^{63}$. En effet, le personnel français de l'Unesco dépasse désormais de près du double le quota de Français théoriquement autorisé64. En 1970, c'est donc à la fois par une nette supériorité numérique du personnel et par une forte influence conceptuelle sur l'orientation et la mise en œuvre des actions de l'Unesco que s'affirme l'influence majeure du domaine francophone au sein de cette organisation.

\section{Conclusion}

Ainsi, les rivalités linguistiques entre l'anglais et le français apparaissent omniprésentes à l'Unesco dans les années 1945-70, à la fois dans les nombreuses assemblées et réunions, dans l'administration, dans la rédaction des textes, rapports, articles et publications, et dans les actions concrètes menées sur le terrain. En outre, au-delà de la dimension linguistique, ces luttes d'influence ont une portée culturelle, politique et économique. Leur enjeu symbolique est très important. L'affirmation croissante du domaine francophone au sein de l'Unesco entre 1945 et 1970 peut s'expliquer à la fois par le rôle individuel des personnalités placées à la tête de l'institution (Torres Bodet et Maheu notamment), à l'importance et au dynamisme des réseaux d'intellectuels francophones qui gravitent autour de l'Unesco, et à l'action volontariste de réseaux diplomatiques et politiques. La prépondérance de la langue et de l'influence française à l'Unesco se poursuivra dans les années suivantes, et ce phénomène sera l'un des facteurs qui amèneront en 1984-85, les États-Unis et le Royaume-Uni à se retirer de l'Unesco.

\section{NOTES}

1. Archives de l'Unesco, X 07.21 (44) D, I : « Délégation de la France»; Roger Caillois, «In memoriam : René Maheu », décembre 1975, p. 10-11.

2. Archives de l'ONU, RAG 2/76, box 10 : lettre de D. Osborne à H. Laugier, 25 nov. 1946 : rapport préliminaire sur la $1^{\text {re }}$ conférence générale de l'Unesco. 
3. Archives de l'Unesco, Unesco and economic development, an adress by Walter H.C. Laves, 10 juin 1953, p. $4:$ « new markets overseas and opportunities for investments ».

4. Archives diplomatiques françaises, NUOI, carton 355 : note confidentielle du 22 novembre 1946.

5. Ronald W. Clark, The Huxleys, London, Heinemann 1968, p. 313.

6. Archives de l'ONU, RAG 2/76, box 10 : rapport confidentiel de Dorothy Osborne à L. Gros, 13 déc. 1946, p. 1-2.

7. Archives diplomatiques françaises, site de Nantes, carton 117 : «La deuxième session du conseil exécutif et le rôle de la France à l'Unesco », 3 août 1947, p. 5-6.

8. Archives diplomatiques françaises, NUOI carton 355: note du ministère des Affaires étrangères, 21 décembre 1946 ;Julian Huxley, Memories II, New York, Harper and Row publishers, 1973, p. 17-18.

9. Archives de l'Unesco, dossier biographique de Jean Thomas.

10. Émile Delavenay, « Mes souvenirs de J. Thomas : tradition 1984, p. 56-62.

11. FR, NUOI carton 333 : note du 3 août 1947 ; Julian Huxley, Memories II, op. cit., p. 44.

12. Archives diplomatiques françaises, site de Nantes, carton $117:$ "La deuxième session ... », doc. cité, p. 13-15.

13. Archives diplomatiques américaines, Decimal file, RG 59, entry CDF 1945-49, box 2242 : lettre de J. Huxley à Walter Laves, 22 sept. 1947.

14. Archives diplomatiques françaises, site de Nantes, carton $117:$ «La deuxième session ... », doc. cité, p. 10, 12, 16-17.

15. Le Figaro, 5 mars 1948, p. 1 et $6:$ « La France à l'Unesco », par André Siegfried.

16. José Luis Martinez, Semblanzas de Académicos, ediciones del centenario de la academia mexicana, México, 1975, 313 p., article sur Torres Bodet; Seth Spaulding et Lin Lin, Historical Dictionary of the UNESCO, The Scarecrow Press, Inc., Lanham, Md., and London, 1997, p. 48 ; Courrier de l'Unesco, décembre 1948, p. 2: «En deux ans, 1200000 Mexicains apprirent à lire. Biographie de M. Torres Bodet».

17. Archives de l'Unesco, DG/161, 7 janvier 1952, p. 11 ; PER/ST/8, p. 2.

18. Lien-Link (bulletin des anciens fonctionnaires de l'Unesco), $\mathrm{n}^{\circ} 80$ : «Jaime Torres Bodet. Thoughts on the one-hundredth anniversary of his birth ", par Alfredo Picasso de Oyague.

19. Mirèse Akar, "Portrait: DG de l'Unesco, René Maheu », L'Orient-Le Jour, n 83, 6-12 janvier 1973, p. 13-15.

20. Archives diplomatiques françaises, Cabinet du ministre : cartons 38 (G. Bidault) et 114 (R. Schuman).

21. Interview de Claude Lévi-Strauss par Chloé Maurel, 17 janvier 2003.

22. Claude Lévi-Strauss et Didier Eribon, De près et de loin, suivi d'un entretien inédit, « Deux ans après ", éditions Odile Jacob, 1990, p. 90.

23. Archives diplomatiques américaines, Decimal file, RG 59, entry CDF 1945-49, box 2254 : rapport confidentiel d'Arthur Compton à G.V. Allen sur la conférence générale de 1948, p. 2.

24. Archives diplomatiques britanniques, FO 371/88915: mémorandum de Paul Matthews au Foreign Office, 26 janvier 1950, intitulé «Unesco», p. 5. «The DG himself considers himself French in sympathy ».

25. Archives diplomatiques américaines, Decimal file: 398.43. RG 59. Department of state, 1950-54, box 1600, lettre confidentielle de K. Holland à Ch. Thomson, 16 janvier 1950, sur les activités de l'Unesco.

26. Le Journal d'Égypte : 4 déc. 1948, $1^{\text {er }}$ déc. 1948, 29 nov. 1948, articles de Marcel Perrier.

27. Archives diplomatiques américaines, box 1566: rapport confidentiel de l'ambassade américaine à Beyrouth au département d'État, 21 oct. 1958.

28. Après l'effondrement en 1918 de l'Empire Ottoman auquel il était soumis, le Liban a été confié à la France comme mandat de la Société des Nations de 1923 à 1944. 
29. Archives diplomatiques françaises, NUOI 1946-1959, carton $n^{\circ} 333$ : télégramme de M. Baudet au ministère des Affaires étrangères, 14 juin 1951.

30. Archives diplomatiques américaines, Decimal file, RG 59, entry CDF 1945-49, box 2243 : lettre confidentielle de W. Benton au Secrétaire d'état, 22 sept. 1947.

31. Archives diplomatiques françaises, NUOI carton 355, GL/AL, 15 décembre 1949, direction générale des relations culturelles, service de l'Unesco: note confidentielle pour le secrétaire général.

32. Archives diplomatiques américaines, box 1601 : rapport confidentiel de K. Holland, 15 mars 1950 ; « the main bulwark of American interest in the organisation. »

33. SC/ADM/12, cf. Gail Archibald, Les États-Unis et l'Unesco, 1944-1963, Paris, Publications de la Sorbonne, 1993, p. 170.

34. É. Delavenay, op. cit., p. 345 ; Peter Lengyel, International Social Science: the Unesco experience, Transaction Books, New Brunswick, USA, et Oxford, 1986, p. 17 ; M. Prévost, L'̂̂le des Uneskimos, Mémoires d'un ancien fonctionnaire de l'Unesco, 1949-1983, janvier 1996, dactylographié, non publié, conservé aux archives de l'Unesco, p. 145.

35. Archives diplomatiques américaines, Decimal file: 398.43. RG 59. Department of state, 1950-54, box 1600 : rapport confidentiel de K. Holland à Ch. Thomson, 6 janv. 1950.

36. Archives diplomatiques américaines, Decimal file, RG 59, entry CDF 1945-49, box 2254 : rapport confidentiel de K. Holland à Ch. Thomson, 21 janv. 1949.

37. Archives diplomatiques américaines, Decimal file: 398.43. RG 59. Department of state, 1950-54, box 1600 : télégramme confidentiel de K. Holland au département d'État, 27 janv. 1950.

38. United Nations Relief and Works Agency for Palestine Refugees in the Near East (UNRWA) : agence des Nations Unies mise en place en 1948 pour fournir de l'aide éducative, des soins médicaux, des services matériels et sociaux et de l'aide humanitaire d'urgence aux réfugiés palestiniens.

39. Archives de l'Unesco, 37 : 362.92 (5-011) «-66», IV : mémo de R. Maheu à McCune, 26 juill. 1961.

40. Archives diplomatiques françaises, Relations culturelles 1951-52. Cuuvres diverses. Carton $n^{\circ}$ 242 : lettre du ministre des affaires étrangères au résident général de France en Tunisie, 20 nov. 1952.

41. L'expérience-témoin d'Haïti, première phase. 1947-1949, Paris, Unesco, 1951 ; et 375 (729.4) A 61, XIX : lettre de McConnell à Adiseshiah, 27 mai 1952 ; lettre de M. Adiseshiah à Wakefield, 10 avril 1952 ; 375 (729.4) A 61, XX : lettre d'A. Lestage au directeur du département de l'éducation, 2 juin 1953 ; et interview d'André Lestage par Chloé Maurel, 24 février 2003.

42. Archives de l'Unesco, 375 (729.4) A 61, XXI : lettre de L. Bernot à A. Lestage, 6 fév. 1954.

43. Elhem Chniti, La Grande-Bretagne et l'Unesco, 1942-1957, 12 ans de relations entre une institution des Nations-Unies et une puissance fondatrice, thèse de doctorat d'histoire, janvier 1997, université Paris I, dir. R. Girault, p. 687-688, 138-139, 258-259.

44. G. Archibald, op. cit., p. 156.

45. Émile Delavenay, Témoignage. D’un village savoyard au village mondial, 1905-1991, Edisud, La Calade, Aix-en-Provence, 1992, p. 373.

46. Émile Delavenay, « Mes souvenirs de J. Thomas », article cité.

47. Gérard Bolla, «Luther H. Evans, librarian et directeur général », in Lien-Link n 82.

48. Archives diplomatiques américaines, box 1566: lettre confidentielle de l'ambassade américaine à Paris au département d'État, 15 octobre 1958, 8 p., p. 3 : « His tendency to call a spade a spade, his rough and ready cantakerousness, his presumed disrespect for things 'Cultural' with a capital $\mathrm{C}$ and his declared preoccupation with the needs of underdeveloped areas have produced considerable resentment and dislike among Western and Southern Europeans. "

49. É. Delavenay, op. cit., p. 373. 
50. Odile Felgine, Roger Caillois, raison et vertiges : un homme dans son siècle, thèse de littérature, Paris IV, 1993, p. 366.

51. É. Delavenay, op. cit., p. 395.

52. É. Delavenay, op. cit., p. 395 ; Archives diplomatiques françaises, NUOI carton 835, note du 16 septembre 1959.

53. Institut Luigi Sturzo (Rome), archives privées de V. Veronese, carton 23 : communiqué de presse $n^{\circ} 2159$, Paris, 2 nov. 1961 : démission du directeur général de l'Unesco; et deux letttres de Veronese au président $\mathrm{du}$ conseil exécutif, Mohamed Awad (l'une publique, l'autre confidentielle), 14 oct. 1961.

54. Archives diplomatiques américaines, Decimal file, RG 59, entry CDF 1960-63, box 820 : lt. confid. de Kellermann au département d'État, 20 juill. 1960; box 823 : mémorandum de conversation entre Maheu et Harlan Cleveland, 5 juill. 1961, p. 1 ; lt. confidentielle de John H. Morrow au département d'État, 18 juill. 1961, intitulée "Who is running Unesco ?»; OHRO, interview de Luther Evans, p. 319 et 699 ; EU, box 825 : mémorandum de Donald B. Eddy à Hefner, 31 mai 1962, 4 p., p. 2-3.

55. Archives diplomatiques britanniques, PREM 11/5185 : record of meeting with Prime Minister, 10 avril 1964 : note confidentielle intitulée : R. Maheu.

56. Archives diplomatiques britanniques, OD 24/15 ; fonds Benton, box 397 : lettre de W. Benton à H. Cleveland et L. Battle, 21 oct. 1963.

Archives diplomatiques françaises, site de Nantes, carton 6, lettre de Jean Fernand Laurent à la DGACT, 21 juill. 1967 ; lettre de Jean-Fernand Laurent au ministre des affaires étrangères, 24 oct. 1967.

57. Archives diplomatiques britanniques, OD 24/041 : rapport de Mary Smieton, juillet 1965, p. 8-9.

58. Archives diplomatiques américaines, subject numeric file, 1970-73, special organizations, box 3225 : airgram de Watson au département d'État, 13 mai 1971, p. 6-7; FR, Nantes, carton 57 : lettre d'H. Costilhes à R. Schuman, 6 mars 1971; X 07.83 Maheu, IV : note sur la coopération entre l'Unesco et la Syrie, 11 janv. 1967, p. 10-11; VI : note sur la coopération entre l'Unesco et la Tunisie, 2 oct. 1974; aide-mémoire sur les entretiens qui ont eu lieu entre les autorités algériennes et le DG de l'Unesco, 3 janv.-5 fév. 1973, p. 2 ; IIa: instructions données par le DG durant sa visite à Alger; EU, box 3225 : airgram de Blake au département d'État, p. 1-3.

59. Archives de l'Unesco, 372 (8) MP01 A 63 (81), III : brochure « CRPE, $3^{\mathrm{e}}$ cours de spécialistes en éducation pour l'Amérique latine, projet majeur n 1 de l'Unesco, 15 mars-10 déc. 1960 ».

60. Archives de l'Unesco, X 07 A 120/197 UNSA : observations et commentaires du gouvernement français sur l'avant-projet de programme et de budget 1965-66, 17 déc. 1963, p. 8-10.

61. Archives de l'Unesco, 3 A 54/53, "Tendances principales de la recherche dans les sciences sociales et humaines» IV : mémo de Julian Hochfeld à Elmandjra, 14 janv. 1966; lettre de M. Elmandjra à J. Hochfeld, 19 janv. 1966.

62. Interview d'Yves Bonnefoy par Chloé Maurel, 16 mars 2004 ; Archives diplomatiques françaises, site de Nantes, carton 104 : lettre de Jean Fernand Laurent au ministre des affaires étrangères, 6 janv. 1970.

63. Archives diplomatiques américaines, Subject numeric file, 1970-73, special organizations, box 3225 : airgram de l'ambassade américaine de Paris au dépar-tement d'État, 24 décembre 1970, p. 44.

64. Archives diplomatiques françaises, cabinet du ministre, Couve de Murville, 180, lettre de M. Couve de Murville à X. Deniau, 25 mai 1968. 


\section{RÉSUMÉS}

Dès la création de l'Unesco en 1945, une concurrence s'y développe entre ses deux langues officielles, l'anglais et le français. Deux groupes d'États aux intérêts linguistiques et culturels divergents s'opposent : le «clan latin » autour de la France, et le « clan anglo-saxon » autour des États-Unis et du Royaume-Uni. La nationalité des dirigeants et du personnel, la langue utilisée dans les réunions et dans les projets de terrain, deviennent l'objet de vives luttes d'influence qui se déploient en marge des enjeux officiels de l'organisation. Au-delà de la dimension linguistique, ces luttes d'influence ont une portée culturelle, politique et économique. De 1945 à 1948, l'Unesco est divisée entre l'influence anglo-saxonne et l'influence francophone. Ensuite, sous la direction du Mexicain francophile Jaime Torres Bodet, de 1948 à 1952, l'influence francophone augmente. Après une tentative de restauration de l'influence anglo-saxonne de 1953 à 1958, la langue française et les conceptions culturelles francophones gagnent du terrain tout au long des années 1960 pour devenir prépondérantes vers 1970. Cette évolution peut s'expliquer à la fois par l'importance et le dynamisme des réseaux d'intellectuels francophones qui gravitent autour de l'Unesco, par l'action volontariste des réseaux diplomatiques et politiques français, et par le rôle individuel des personnalités placées à la tête de l'Unesco, notamment le Français René Maheu pendant les années 1960.

From the creation of Unesco in 1945, a competition develops between its two official languages, French and English. Two groups of States arise, with opposite linguistic interests: the "Latine clan" (around France) and the "Anglo-Saxon clan" (around the United States and the United Kingdom). The nationality of staff members, the language used in meetings and field projects, become controversial. These struggles of influence have not only a linguistic dimension, but also a cultural, political and economic dimension. From 1945 to 1948, Unesco is divided into Englishspeaking and French-speaking influence. Then, under the management of the francophile Mexican Jaime Torres Bodet from 1948 to 1952, the French influence grows. From 1953 to 1958, Anglo Saxons try to restore their influence on Unesco. During the 1960', French influence grows and becomes dominant around 1970. This evolution explains itself by the importance of the French-speaking intellectual networks, by the action of political and diplomatic French networks, and by the individual role of Directors of Unesco such as the Frenchman René Maheu in the 1960'.

\section{INDEX}

Mots-clés : francophonie, Unesco, guerre froide, anglophonie, diplomatie, culture

Keywords : francophony, Unesco, Cold war, anglophony, diplomacy, culture

\section{AUTEUR}

\section{CHLOÉ MAUREL}

Chercheuse associée à l'IHTP, au CHCSC de l'université de Versailles Saint-Quentin-en-Yvelines, à l'IHMC et à l'IRICE chmaurel@yahoo.fr 